\title{
A Virtual Reality App for Physical and Cognitive Training of Older People With Mild Cognitive Impairment: Mixed Methods Feasibility Study
}

Mary Hassandra $^{1 *}, \mathrm{PhD}$; Evangelos Galanis ${ }^{1 *}, \mathrm{PhD}$; Antonis Hatzigeorgiadis ${ }^{1 *}, \mathrm{PhD}$; Marios Goudas ${ }^{1}, \mathrm{PhD}$; Christos Mouzakidis $^{2}$, PhD; Eleni Maria Karathanasi², BSc; Niki Petridou², BSc; Magda Tsolaki², ${ }^{2,3}$ PD, MD; Paul Zikas ${ }^{4}$, $\mathrm{PhD}$; Giannis Evangelou ${ }^{4}$; George Papagiannakis ${ }^{4,5}, \mathrm{PhD}$; George Bellis ${ }^{6}$, BEng; Christos Kokkotis ${ }^{1,6}$, MSc, BEng; Spyridon Rafail Panagiotopoulos ${ }^{6}$, BEng; Giannis Giakas ${ }^{1}, \mathrm{PhD}$; Yannis Theodorakis ${ }^{1}, \mathrm{PhD}$

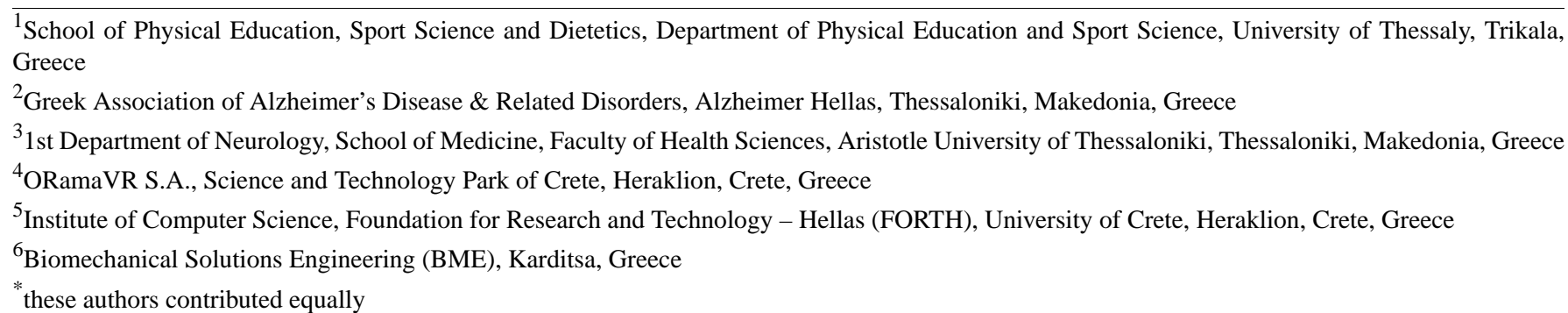

Corresponding Author:

Mary Hassandra, $\mathrm{PhD}$

School of Physical Education, Sport Science and Dietetics

Department of Physical Education and Sport Science

University of Thessaly

Karies

Trikala, 42100

Greece

Phone: 30243104700

Email: mxasad@uth.gr

\section{Abstract}

Background: Therapeutic virtual reality (VR) has emerged as an effective treatment modality for cognitive and physical training in people with mild cognitive impairment (MCI). However, to replace existing nonpharmaceutical treatment training protocols, VR platforms need significant improvement if they are to appeal to older people with symptoms of cognitive decline and meet their specific needs.

Objective: This study aims to design and test the acceptability, usability, and tolerability of an immersive VR platform that allows older people with MCI symptoms to simultaneously practice physical and cognitive skills on a dual task.

Methods: On the basis of interviews with 20 older people with MCI symptoms (15 females; mean age 76.25, SD 5.03 years) and inputs from their health care providers (formative study VR1), an interdisciplinary group of experts developed a VR system called VRADA (VR Exercise App for Dementia and Alzheimer's Patients). Using an identical training protocol, the VRADA system was first tested with a group of 30 university students ( 16 females; mean age 20.86, SD 1.17 years) and then with 27 older people (19 females; mean age 73.22, SD 9.26 years) who had been diagnosed with MCI (feasibility studies VR2a and VR2b). Those in the latter group attended two Hellenic Association Day Care Centers for Alzheimer's Disease and Related Disorders. Participants in both groups were asked to perform a dual task training protocol that combined physical and cognitive exercises in two different training conditions. In condition A, participants performed a cycling task in a lab environment while being asked by the researcher to perform oral math calculations (single-digit additions and subtractions). In condition B, participants performed a cycling task in the virtual environment while performing calculations that appeared within the VR app. Participants in both groups were assessed in the same way; this included questionnaires and semistructured interviews immediately after the experiment to capture perceptions of acceptability, usability, and tolerability, and to determine which of the two training conditions each participant preferred. 
Results: Participants in both groups showed a significant preference for the VR condition (students: mean 0.66, SD 0.41, $t_{29}=8.74, P<.001$; patients with MCI: mean 0.72, SD 0.51, $\left.t_{26}=7.36, P<.001\right)$, as well as high acceptance scores for intended future use, attitude toward VR training, and enjoyment. System usability scale scores $(82.66$ for the students and 77.96 for the older group) were well above the acceptability threshold (75/100). The perceived adverse effects were minimal, indicating a satisfactory tolerability.

Conclusions: The findings suggest that VRADA is an acceptable, usable, and tolerable system for physical and cognitive training of older people with MCI and university students. Randomized controlled trial studies are needed to assess the efficacy of VRADA as a tool to promote physical and cognitive health in patients with MCI.

(JMIR Serious Games 2021;9(1):e24170) doi: 10.2196/24170

\section{KEYWORDS}

virtual reality; elderly; mild cognitive impairment; combined physical and cognitive function; dual task

\section{Introduction}

\section{Background}

With an aging population across developed countries, ensuring an independent and healthy lifestyle for older people has become a key social issue and a global public health priority [1]. In this regard, the World Health Organization (WHO) has called for more research to identify ways to support the needs of people living with dementia. According to the WHO, "dementia is a syndrome, of a chronic or progressive nature, in which there is deterioration in cognitive function beyond what might be expected from normal aging." The condition affects memory, thinking, orientation, comprehension, calculation, learning capacity, language, and judgment. Impaired cognitive function is commonly accompanied or preceded by deteriorating motivation, emotional control, or social behavior. At any given time, an estimated $5 \%$ to $8 \%$ of people aged 60 years and above have dementia; the global total is projected to reach 82 million by 2030 and 152 million by 2050 .

Alzheimer disease (AD) is the most common form of dementia and accounts for $60 \%$ to $70 \%$ of cases [2]. This is often preceded by a predementia stage known as mild cognitive impairment (MCI), which is an intermediate state between normal aging and dementia involving memory deficits [3] and difficulties with language, thinking, and judgment beyond normal age-related changes that may not be obvious in everyday activities. MCI may be amnesic, nonamnesic, or single- or multiple-domain. The amnesic type includes memory loss and is regarded as a transition stage between normal aging and AD [4]. Studies suggest that 5\%-20\% of individuals with MCI will develop dementia each year [5,6]. Although MCI does not meet the criteria for dementia, it affects healthy aging and indicates a possible need for medical care and treatment. As all forms of degenerative dementia are incurable, treatment focuses primarily on slowing its progression and managing symptoms, typically through a combination of medication and lifestyle changes $[7,8]$.

\section{Physical and Cognitive Training}

Among nonpharmacological treatments for MCI, combined physical and cognitive training programs are now gaining wider acceptance as part of the standard treatment for people with symptoms of dementia [5,9-11]. Research suggests that physical and cognitive development are interdependent and closely related [12-17], probably because neurogenesis continues even in older adulthood [18,19], and physical exercise is a key dose-related facilitator of neurogenesis [20,21]. New experiences provided by systematic and intense exercise promote alterations in the brain that can contribute to cognitive rehabilitation. Recent studies have reported improved cognitive performance following combined physical and cognitive activities compared with either one alone [22]. This suggests that simultaneous execution of cognitive and motor tasks may yield the greatest improvements in cognitive function [23]. This dual task testing requires significant cognitive control of attentional and executive functions and helps to identify patients with MCI who are at high risk of developing dementia [24]. Dual task training has been tested in dementia intervention studies $[23,25,26]$ and has shown promise in patients with neurological disorders (including $\mathrm{MCI}$ ) as a means of improving balance [27], gait, and cognitive ability [28]. Preliminary evidence also suggests that direct and indirect interventions targeting cognitive-motor interference can help older people with neurodegenerative diseases [29].

Early diagnosis of MCI, including subtype and stage, facilitates earlier treatment and care that can minimize the onset of neurodegeneration, optimize cognitive and physical functioning, and improve quality of life. Emerging technologies offer novel options for research and practice; among these, virtual reality (VR) is a valuable addition as a safe and controlled environment for user interaction and monitoring of physical activity and cognitive task performance. Manipulation of experimental parameters in VR apps has great potential for new forms of dementia intervention and treatment [30-32].

\section{VR for Combined Physical and Cognitive Training}

VR has shown potential as a tool for assessing and training patients diagnosed with dementia and MCI [33,34]. According to recent reviews, VR-based training interventions can be used to improve well-being, cognition, and physical fitness in people with MCI [30,35,36]. Among recent VR systems combining physical training and cognitive training [37,38], the system by Mrakic-Sposta et al [37] involved sequential tasks of riding a bike in a park and avoiding cars while crossing the road to a supermarket. When the system was tested on 10 older patients with MCI and a control group, the results indicated some improvement in cognitive functions, including visual-constructive, visuo-spatial attention, executive, and memory functions, as well as verbal fluency. However, none of the changes was statistically significant [37]. In a more recent 
randomized controlled trial (RCT) [38], a 12-week VR-based physical and cognitive training program led to significant improvements in dual task gait performance among older people with MCI. The physical training elements included Tai Chi, resistance and aerobic exercises, and functional tasks such as window cleaning and goldfish scooping. The cognitive elements included VR games such as buying tickets from vending machines, finding items in a virtual store, and preparing meals as a kitchen chef. Both of these dual task systems for patients with MCI [37,38] were sequential rather than simultaneous. Although closer to everyday situations, these programs also involved multiple cognitive tasks, and some were difficult to compare with standard treatment. In addition, the equipment is expensive and complicated and requires trained specialists to make decisions about system settings and special guidance for participants to ensure efficient training.

On the basis of earlier studies, we designed a simultaneous dual task VR system for physical and cognitive training to support people with MCI. Drawing on clinical findings regarding MCI rehabilitation and new VR technology, we adopted a person-centered approach to develop a user-friendly, immersive VR training system called VRADA (VR Exercise App for Dementia and Alzheimer's Patients). The program content was based on previously tested combined protocols for physical and cognitive therapy [39]. In addition, the VRADA exercises allow for future comparisons with standard care and training protocols.

\section{Design of VR Training Environments}

Immersive VR enables researchers to create realistic environments while maintaining a high level of experimental control of essential elements, such as visual and audio feedback and virtual characters. The experience of a virtual body in an immersive virtual environment is similar to the sensations of the biological body [40], and there is a profound link between embodiment and learning [41]. The concept of presence refers to the phenomenon of behaving and feeling as if we are actually in the virtual world. This powerful sensation is unique to VR and cannot be created in any other medium. Most people find this magical; unlike immersion, where the user is simply surrounded by digital screens, VR enriches immersion and embodiment in a realistic multisensory environment.

Research on the learning impact of embodiment in virtual multimodal environments [42] shows that these settings can facilitate skill transfer when deployed realistically. As embodied navigation and memory are closely linked [43], virtual promenades can compensate for reduced spontaneous motion in older people [44]. In general, embodiment is valuable in these training scenarios because the motivation to experience actions that the situation demands links the user psychologically to the virtual world. VR technology facilitates mixed-ability learning and knowledge transfer and helps participants to interact and collaborate fruitfully through different modalities, and there is evidence that immersive virtual environments enhance training in motor and spatial activities $[45,46]$.

\section{Person-Centered Approach}

According to the Alzheimer Society, a person-centered approach is strongly encouraged in dementia care settings, tailoring care to the individual's interests, abilities, history, and personality [47]. This umbrella term is used in different disciplines to describe a model that promotes personal autonomy or self-determination within one's environment. On the basis of self-determination theory, this approach emphasizes the importance of understanding the motivation that drives a person's behavior and the extent of that motivation. The distinction between autonomous and controlled forms of motivation is central to the theory $[48,49]$.

Neurocognitive disorders are commonly associated with symptoms of apathy and are expressed as low motivation and interest in daily activities, which are known to increase the risk of progression from $\mathrm{MCI}$ to $\mathrm{AD}[50,51]$. Therefore, encouragement and motivation are important components of every training program. To address the issue of low motivation to exercise and adherence to training among patients with MCI, we incorporated the following motivational techniques [52] in the VRADA training system. These are derived mainly from self-determination theory [53,54].

- Goal setting: choices for exercise duration when starting each training session

- Feedback on behavior: informative or evaluative feedback at the end of each session on training performance (total distance, cycling time, and number of correct answers on cognitive exercises)

- $\quad$ Task crafting (enjoyment): choice of music to enjoy during training

- Self-monitoring of behavior: screen displays indicating time, speed, and distance for monitoring performance while exercising

The VRADA system design is based on human-centered design, which is a systematic method for developing usable products, systems, or services by focusing explicitly on the intended user [55]. The goal is to maximize relevance and usability, which are defined as the extent to which specified users can use a product to achieve specified goals effectively, efficiently, and with satisfaction in a specified context of use. There is evidence that involving users in the design and development of a new system will improve the system's quality by ensuring a more accurate assessment of user requirements and a higher level of user acceptance [56]. This study followed these design principles, strategies, and recommended best practices for formative and feasibility studies [57].

\section{Objectives}

This study has 2 main objectives: (1) to describe the formative study of the VRADA system, focusing on how content was developed by working with patients and health care providers and applying the principles of human-centered design and continuous testing (VR1) and (2) to report 2 studies assessing the acceptability, usability, and tolerability of the VRADA system among a sample of university students and a sample of older people with MCI (VR2a and VR2b). 


\section{Methods}

\section{Formative Study VR1}

In designing the VRADA system, we adopted a human-centered approach by involving patients (as future users) from the early stages, conducting individual interviews to learn about their relevant needs, experiences, attitudes, beliefs, preferences, aspirations, and expectations, and documenting diverse opinions by gender, education level, and cognitive disorder. From the outset of project planning, we also involved health professionals from the Alzheimer Hellas Day Centers, which are responsible for patients' physical and cognitive training in order to consult with them during the design, application, and evaluation phases of the VRADA project.

\section{Study Aim}

The aim of this study is to collect information about patients' training experiences, preferences, and expectations to develop a user-centered training system.

\section{Participants and Setting}

Patients were recruited for interviews at 2 Hellenic Alzheimer Association Day Centers in Thessaloniki in August 2018. Older people visit these centers voluntarily for neuropsychological assessments, neurological examinations, and case management. A total of 20 patients volunteered to participate in the study (15 women and 5 men; mean age 76.25, SD 5.03 years; age range 69-84 years). In total, 16 had been diagnosed with MCI and 4 with subjective cognitive decline; most patients were retired (mean years of education 11.35, SD 5.76; range 5-18 years).

\section{Assessments}

The 2 trained psychologists who worked at the 2-day care centers conducted the interviews using an open-ended questionnaire as a guide. The interview topics were type, frequency, and duration of exercise sessions the interviewee attended at the day care center and whether they engaged in any additional physical activity elsewhere; preferred outdoor exercise environment (scenery, season, time of day, social environment, and music); feedback options while exercising (speed, time, distance, and heart rate); standing bike preferences (familiarity with biking and sitting bike vs upright bike); and cognitive exercises they would most enjoy while biking (memory, attention, and problem solving).

\section{Procedure and Data Analysis}

Two interviewers invited older people to participate voluntarily in the study. The first 20 who accepted then signed informed consent forms, and all participants agreed to the use of a voice recorder. Interviews lasted for 30 minutes to 1 hour and were subsequently transcribed verbatim for thematic analysis of each topic or question. All answers were organized in tabular format, and similar answer frequencies were calculated.

\section{Feasibility Studies VR2a and VR2b}

\section{Study Aim}

In the 2 identical test studies with university students (VR2a) and end users (VR2b), we focused on the acceptability, usability, and tolerability of using the VRADA system as compared with standard care physical and cognitive training.

\section{Participants}

A total of 30 undergraduate students (14 males and 16 females) from a physical education and sports science department participated in study VR2a. The mean age of the participants was 20.86 years (SD 1.17). In study VR2b, 27 participants ( 8 males and 19 females) were recruited from 2-day care centers run by the Greek Association of Alzheimer's Disease and Related Disorders - Alzheimer Hellas. The latter group comprised patients who had been diagnosed with MCI according to the Petersen criteria [58] and were at stage 3 of the disease according to the Global Deterioration Scale [59], with a Clinical Dementia Rating [60] score of 0.5 and exhibiting subjective cognitive decline [61]. They ranged in age from 59 to 85 years (mean age 73.22, SD 9.26 years). Demographic details (educational level, exercise habits, and use of technology) are presented in Multimedia Appendix 1.

\section{Apparatus}

A cycle-ergometer (stationary seated bike type; Toorx, Chrono Line, BRX R 300) was identified as the optimal choice for the exercise apparatus, as it has been proven to reduce user fall risk and facilitate precise control of training conditions. It also meets the requirements of Bluetooth connectivity capability.

\section{Application Description}

This section details the functionalities and phases of the VR apps used (a demo video is available in Multimedia Appendix 2 ). On first running the VRADA app, the user must select the number of minutes they aim to cycle within the virtual environment. As a selection mechanism, we implemented a raycast from the VR controller, allowing the user to select an answer by pointing the ray at the button and pressing the trigger button on the controller (Figure 1). 
Figure 1. User selects the daily goal for the duration of the training session.

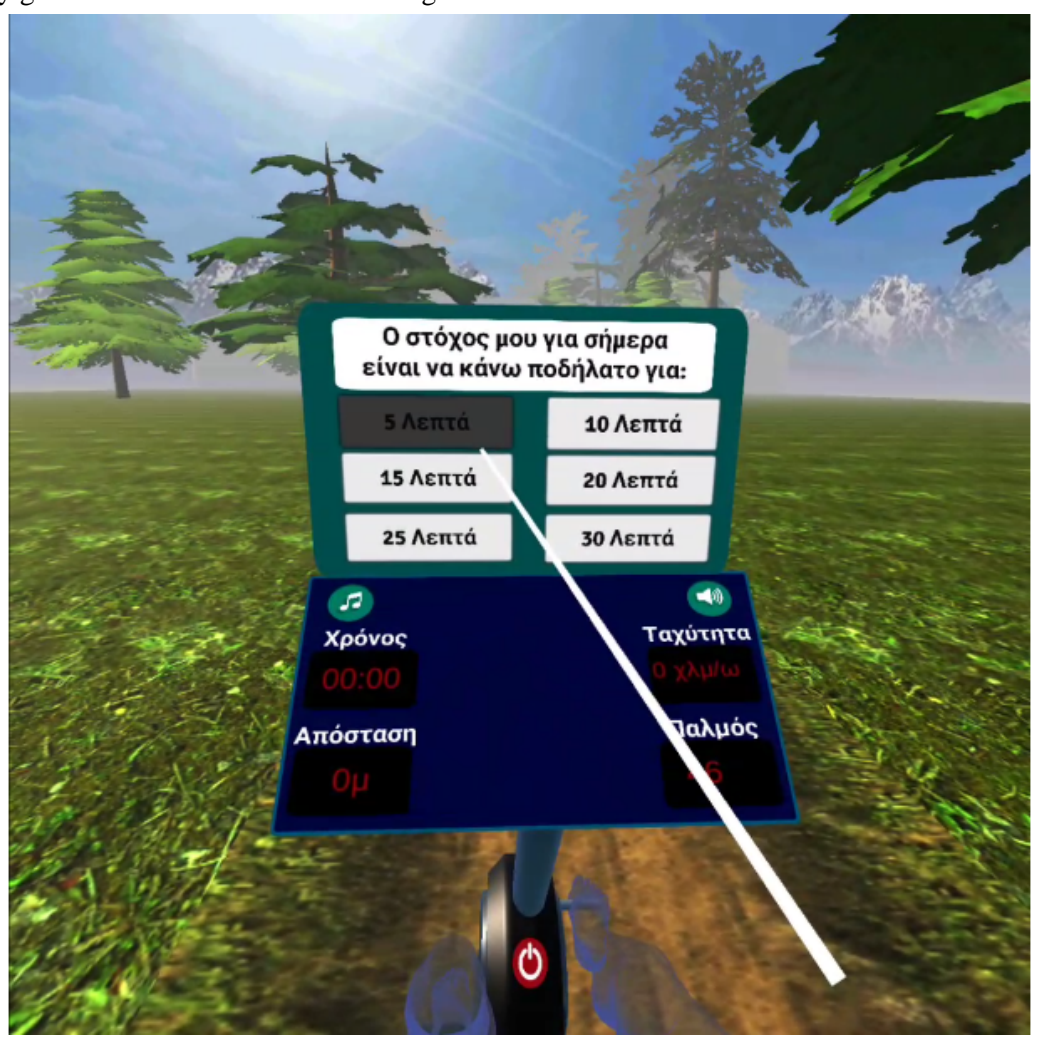

At this point, the user can begin cycling on the training bike. During cycling, the user can choose to listen to music from a list of preloaded tracks. The images below (Figure 2) show the

math quiz, which asks the user to complete a simple subtraction of 2 single-digit numbers. In this example, as the user answered correctly, the answer is highlighted in green.

Figure 2. User selects the answer to the math calculation exercise.

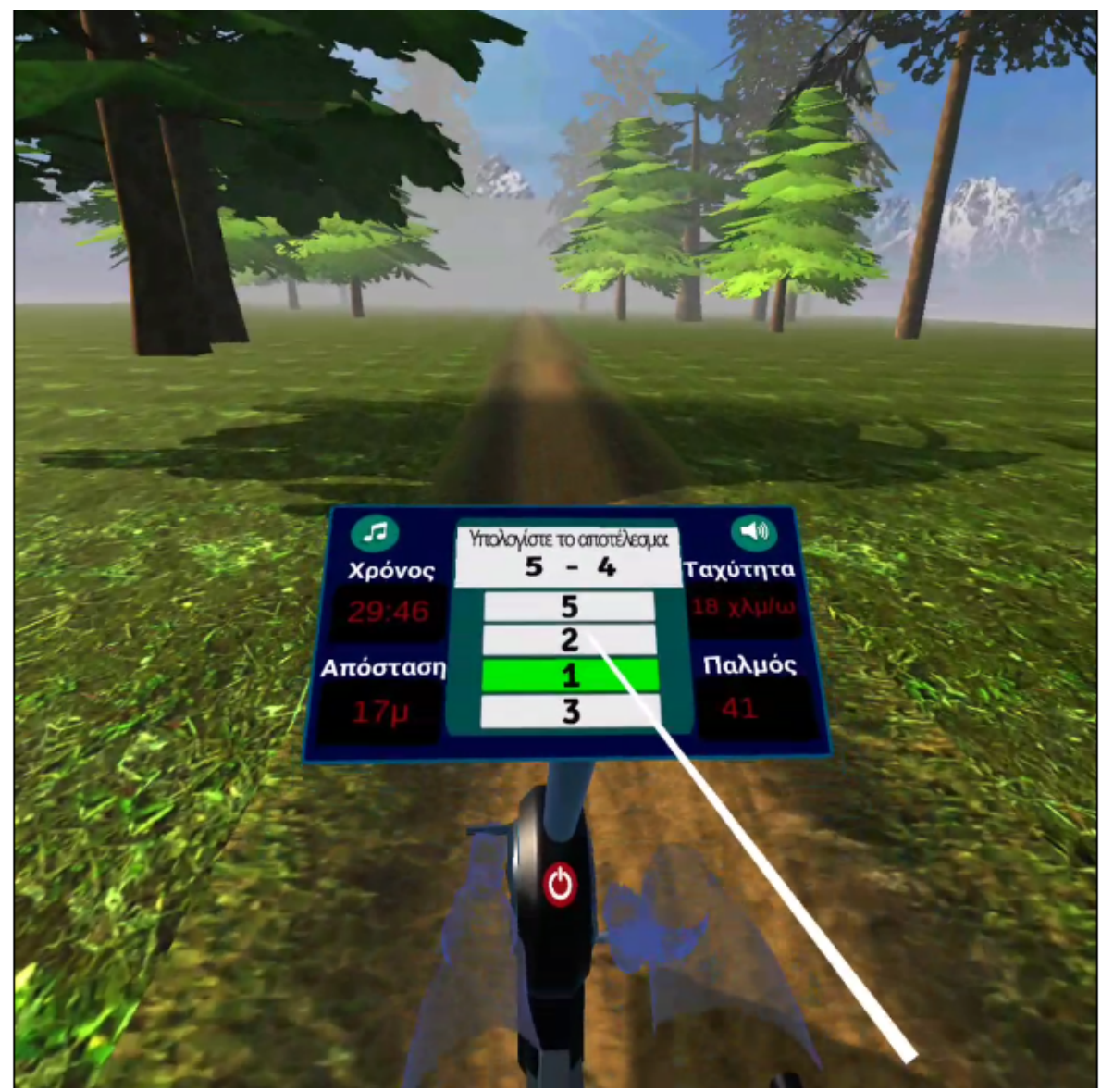


After completing the cycling session, the user analytics were displayed in front of the bike, identifying correct and incorrect answers from the math quiz. Finally, the user was asked to evaluate their performance and report any difficulties (Figure $3)$.

Figure 3. Users' closing screen after completion of the session.

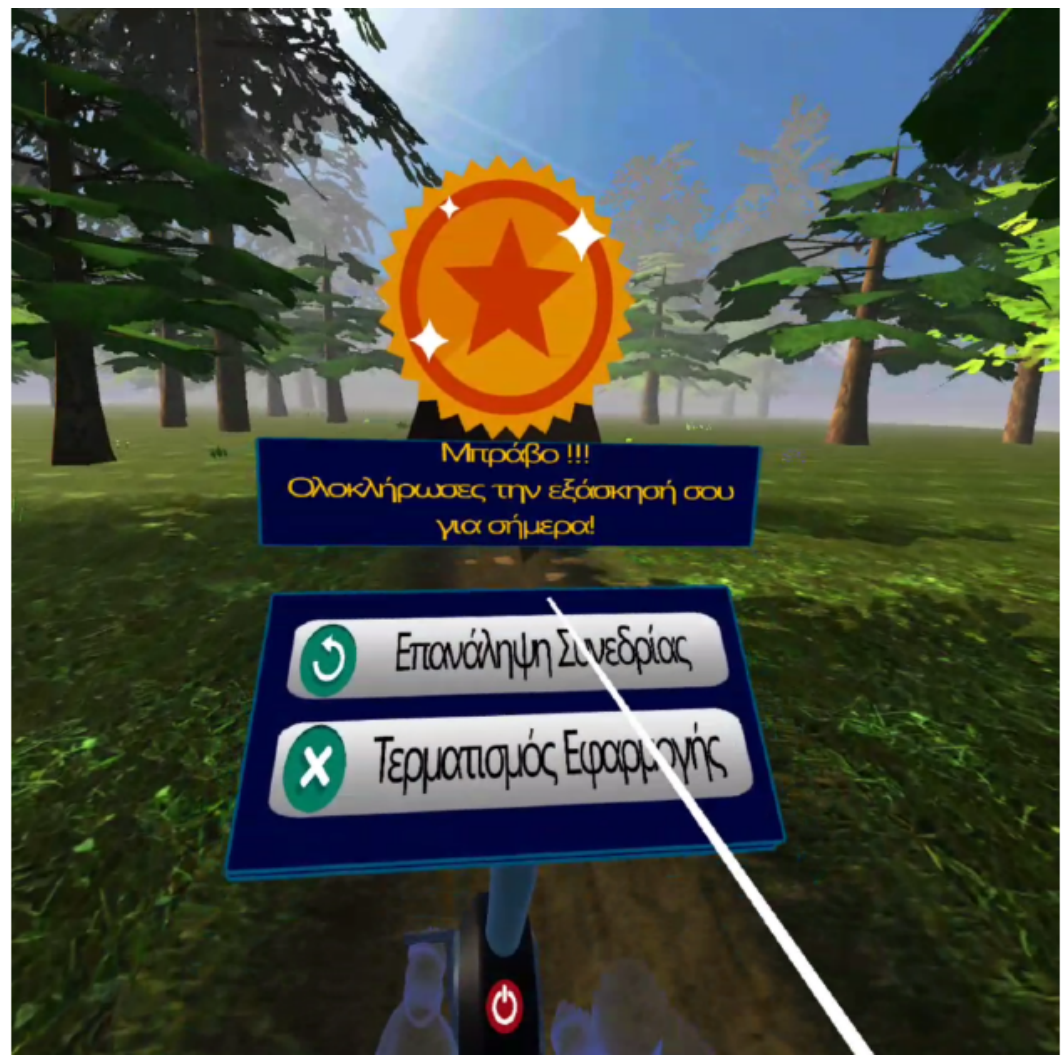

It is important to mention that user data were tracked and exported in an analytics file at the end of each session. This file, containing the answers from the math quiz and user-stated preferences, was also saved to the headset.

\section{Task and Conditions}

Each participant was asked to perform a dual task protocol combining physical and cognitive tasks under 2 different training conditions. The physical aspect of the task involved cycling (on a stationary seated bike) for 15 consecutive minutes at a constant speed of $15 \mathrm{~km} / \mathrm{h}$. In every trial, the bike workload was initially self-selected by the user and dynamically adjusted by the supervisor in accordance with the exercise protocol. The cognitive task required participants to complete 20 simple numerical calculations (single-digit additions and subtractions) during cycling. In one condition, participants had to execute the cycling task in the lab environment and were asked by the experimenter to perform the calculations orally. In the other condition, participants were required to execute the cycling task in the virtual environment and were asked to perform the calculations that appeared in the VR app using a remote control.

\section{Measures}

\section{Personal Innovativeness}

Personal innovativeness was measured by using 4 items assessing an individual's general tendency to try out new information technologies [62] adopted from Yusoff et al [63] (eg, I am the kind of person who looks forward to experimenting with new technologies). Responses on a 5-point Likert scale ranged from 1 (strongly disagree) to 5 (strongly agree).

\section{Acceptance}

Participants' acceptance of the VRADA app was assessed in terms of 3 factors: perceived enjoyment, attitude, and intended future use. Perceived enjoyment was measured by 6 items that assessed feelings of pleasure while exercising (eg, I really enjoyed exercising in the VR environment) [63]. Responses on a 5-point Likert scale ranged from 1 (strongly disagree) to 5 (strongly agree). Attitudes toward the VRADA app were assessed on the basis of guidelines from planned behavior theory [64] involving 6 bipolar items (eg, pleasant-unpleasant, useful-useless) and scored on a 7-point semantic differential scale. Items assessing intended future use were adapted from a previous study [63] and modified according to the guidelines for assessment of attitudes based on the theory of planned behavior [65]. Three items evaluated the extent to which a person had formulated a conscious plan to exercise in the future in the VR environment (eg, assuming I have access to the system, I intend to use it). Responses on a 5-point Likert scale ranged from 1 (strongly disagree) to 5 (strongly agree).

\section{Usability}

The system usability scale (SUS) [66] was used to assess subjective components of usability. The SUS is a self-report questionnaire comprising 10 items -5 positive (eg, I think that I would like to use this system frequently) and 5 negative (eg, I found the system unnecessarily complex). Responses were 
rated on a 5-point Likert scale ranging from 1 (strongly disagree) to 5 (strongly agree).

\section{Preference}

Preference for the 2 exercise protocols was assessed by 8 questions about exercising in the natural or the virtual environment (eg, Exercise was more pleasant..., the numerical calculations were more fun..., time passed quicker..., when exercising with or without the VR mask). Responses were dichotomous, scoring -1 (against VR) or +1 (in favor of VR).

\section{Additional Assessments}

A questionnaire and semistructured interviews were conducted to collect additional information immediately following the session. The questionnaire [37] included 9 questions assessing participants' perceptions of using the Oculus Go headset and controller and the environment in terms of (1) usability-pleasantness (4 items; eg, I felt comfortable using the mask; I enjoyed the park ride); (2) usability-learning, (2 items, eg, It was easy to read the numerical questions); and (3) tolerability ( 3 items assessing dizziness, boredom, and anxiety). Responses on a 5-point Likert scale ranged from 1 (strongly disagree) to 5 (strongly agree). The semistructured interview [67] collected qualitative information to further explore participants' subjective perceptions and feelings regarding reasons to use VRADA (eg, Why would you use the VRADA training system?); expectations after the session (2 items; eg, given the opportunity, would you be willing to use this training system regularly?); usability or utilization (5 items; eg, What difficulties did you encounter during the training session?); usability or learning ( 2 items; eg, Did you have to ask for help to be able to use the system? Where exactly?); usability or pleasantness ( 2 items; eg, What exactly did you like most and least?); sense of presence or spatial presence ( 2 items; eg, Did you feel you had control over the environment?); sense of presence or engagement (2 items; eg, Did you get distracted during exercise? By what?), sense of presence or realism (How did you find the environment-realistic or too artificial?); and tolerability ( 2 items; eg, Did you feel bad during exercise? When and where exactly?). A detailed interview guide can be found in Multimedia Appendix 3.

\section{Procedure}

As volunteers, participants signed consent forms after being provided with an information sheet describing the study requirements and confirming their right to withdraw from the study at any time. In the preparatory phase of the experimental session, participants were told about the procedure and were encouraged to ask questions. They were then equipped with a VR mask and remote control and were allowed some time to familiarize themselves with this equipment. They were then brought to the stationary bike to make seat adjustments and to become familiar with the task, which involved cycling for 2 minutes. Two training conditions were implemented to complete this preparatory phase. The order of the conditions was counterbalanced, with a 10-minute break between the 2 conditions. Finally, after a 5-minute rest, the participants completed the questionnaire and discussed the interview questions with the experimenter. The entire procedure took approximately 60 minutes to complete.

\section{Data Analysis}

Quantitative data were analyzed using SPSS Statistics version 21 (IBM Corporation). Summary statistics were calculated for demographic characteristics, and correlations among all examined variables were assessed using the Pearson coefficient. Exercise protocol preference was examined using a single-sample two-tailed $t$ test, using $P<.05$ (two-sided) to determine statistical significance. The qualitative interview data were analyzed using thematic analysis [68], which can offer rich insights into attitudes and beliefs by identifying patterns of ideas or responses. As the discussion topics were based on relevant previous studies, the main themes were predetermined (deductive approach). Second-order themes were analyzed using an inductive approach, allowing the data to determine subthemes.

\section{Ethics Approval and Consent to Participate}

The institution's ethics committee granted permission for these studies (approval number: 1557, October 2, 2019). The confidentiality of private personal and health information will be ensured in line with regulations (European Union, EU) 2016/679 (General Data Protection Regulation). Participants were briefed verbally, face-to-face, and provided written information, including the consent form. Where necessary, participants were provided with additional information about the study.

\section{Results}

\section{Formative Study VR1}

Table 1 summarizes the participants' answers regarding their past experiences of physical activity, along with their preferences and expectations for a VR system combining physical and cognitive training.

The results were presented to the research group, comprising providers of patients' physical and cognitive training, their neuropsychiatrist (extensive clinical and research experience in dementia), exercise psychologists (specialists in exercise motivation), a biomedical engineer, and a computer scientist specializing in computer graphics and extended reality. On the basis of their expertise and the input from patients with MCI, the group made collaborative decisions on the design and content of the first VRADA prototype. 
Table 1. Experiences, preferences, and expectations of patients with mild cognitive impairment regarding a virtual reality training environment.

\begin{tabular}{ll}
\hline Topic & Values \\
\hline $\begin{array}{l}\text { Current physical activity } \\
\text { Type of exercise, } \mathbf{n}(\%)\end{array}$ \\
$\quad$ Full body exercises & $15(75)$ \\
$\quad$ Neck and shoulder exercises & $3(15)$ \\
$\quad$ Walking & $2(10)$ \\
Frequency of exercise (times/week), mean (SD) & $2.70(1.55)$ \\
Duration of exercise (min/training), mean (SD) & $53.75(11.57)$
\end{tabular}

\section{Ideal exercise environment}

Scenery, n (\%)

Forest or park

Seaside

Town

Season, n (\%)

Spring

Autumn

Winter

2 (12)

Summer

$1(6)$

Time of day, n (\%)

Morning

Night

Social environment ( exercise with other), n (\%)

Yes

No

Natural environment, n (\%)

Sounds of nature

Birdsong

Waves splashing

Other

Music, $\mathbf{n}(\%)$

Soft classic music

Traditional Greek music

No music

Feedback during exercise

Time, speed, distance, and heart rate, n (\%)

Yes

No

Feedback presentation, $n$ (\%)

Monitor

From training provider

$10(50)$

\section{Type of bike}

Familiar with bike, n (\%)

Yes 


\begin{tabular}{ll}
\hline Topic & Values \\
\hline No & $4(20)$ \\
Balance on stationary bike, $\mathbf{n}(\boldsymbol{\%})$ & $16(80)$ \\
Yes & $4(20)$ \\
No & \\
Type of bike, $\mathbf{n}(\%)$ & $16(80)$ \\
Seated bike & $4(20)$
\end{tabular}

Cognitive exercises

Number calculations, n (\%)

Yes

No

First letter task, n (\%)

Yes

No

Anagrammatical task, n (\%)

Yes

No

Synonyms-antonyms task, n (\%)

Yes

No

Missing words task, $\mathbf{n}(\%)$

Yes

No

Create sentences task, $\mathbf{n}(\%)$

Yes

No

\section{Building the VRADA Training System}

On the basis of information from participant interviews, health professionals from Alzheimer Hellas Day Care Centers, and experts from the exercise psychology research group, the ORAMA-VR team prepared the first prototype, and the biomechanical solutions engineering team incorporated the VRADA software into the bike (Toorx, Chrono Line, BRX R 300). The development procedure was based on continuous testing feedback; that is, each software version was user-tested to inform further development of the prototype. As we felt this might prove burdensome for the intended end users (ie, older people with $\mathrm{MCI}$ ), university students tested the prototype and provided the necessary feedback.

During this period, a bridge device was used to connect the VRADA software to the bike. Other tasks performed during this period included continuous improvement of the VRADA visual environment, introduction and thorough testing of cognitive exercises, construction of data storage and extraction mechanisms, and adjustments to regulation of real and virtual speeds. Throughout this continuous testing period, student users were asked open questions about specific aspects of the development process - for example, alignment of bike pedaling speed with the VR biking experience. Similarly, when adding cognitive exercises to the VR environment, feedback was collected from a series of trials to improve the design and ensure smooth alignment with the VR biking experience.

\section{System Architecture}

The VRADA app was built on top of the ORamaVR MAGES platform [69,70], using the latter's training and interaction mechanics. The MAGES platform is fully customizable and supports educational VR simulations with minimal adaptation. This is accomplished by prototyping the learning pipeline into structured, independent, and reusable segments, which are used to generate more complex behaviors. The architecture supports all current and forthcoming VR head-mounted displays and standard 3-dimensional content generation. The MAGES platform includes the following novel features [70]:

- Multiplayer with geometric algebra interpolation: custom low-bandwidth and high visual fidelity collaborative modules. Our geometric algebra framework enables 4 times the improvement in reduced data network transfer and lower processor usage.

JMIR Serious Games 2021 | vol. 9 | iss. 1 | e24170 | p. 9 (page number not for citation purposes) 
- Analytics based on machine learning agents with recommendations: We used medical experts to train our machine learning agent and constructed a unique trainee profile to make real time suggestions to users according to their level of experience. Our supervised machine learning model is capable of understanding the validity of each action and deciding whether to offer assistance in the form of additional audio-visual guidance.

- Geometric algebra deformable animation, cutting, and tearing: The use of quaternions and dual quaternions yielded fast results, with no interpolation problems or other geometric artifacts. Our engine also performs animations with fewer intermediate keyframes, thereby reducing the bandwidth.
- Editor in VR: This module allows non-VR experts to develop new modules or scenarios or to modify existing ones in a coding-free environment.

- Semantically annotated bodies: The MAGES core includes an advanced mathematical algorithm for physics-based visual techniques that can generate a virtual representation of the body, which is essential for VR physical training.

\section{Virtual Environment}

We designed a forest path as a scenery for a relaxing and enjoyable virtual environment. The forest is dynamically generated as the user cycles along the path (Figure 4). We implemented this mechanism to optimize performance, as the app is deployed on a mobile VR headset, and its performance is limited by the onboard graphics chip. In addition, we populated the forest with animals that the user must remember for the purposes of the memory game at the end of the session.

Figure 4. The dynamically generated forest.

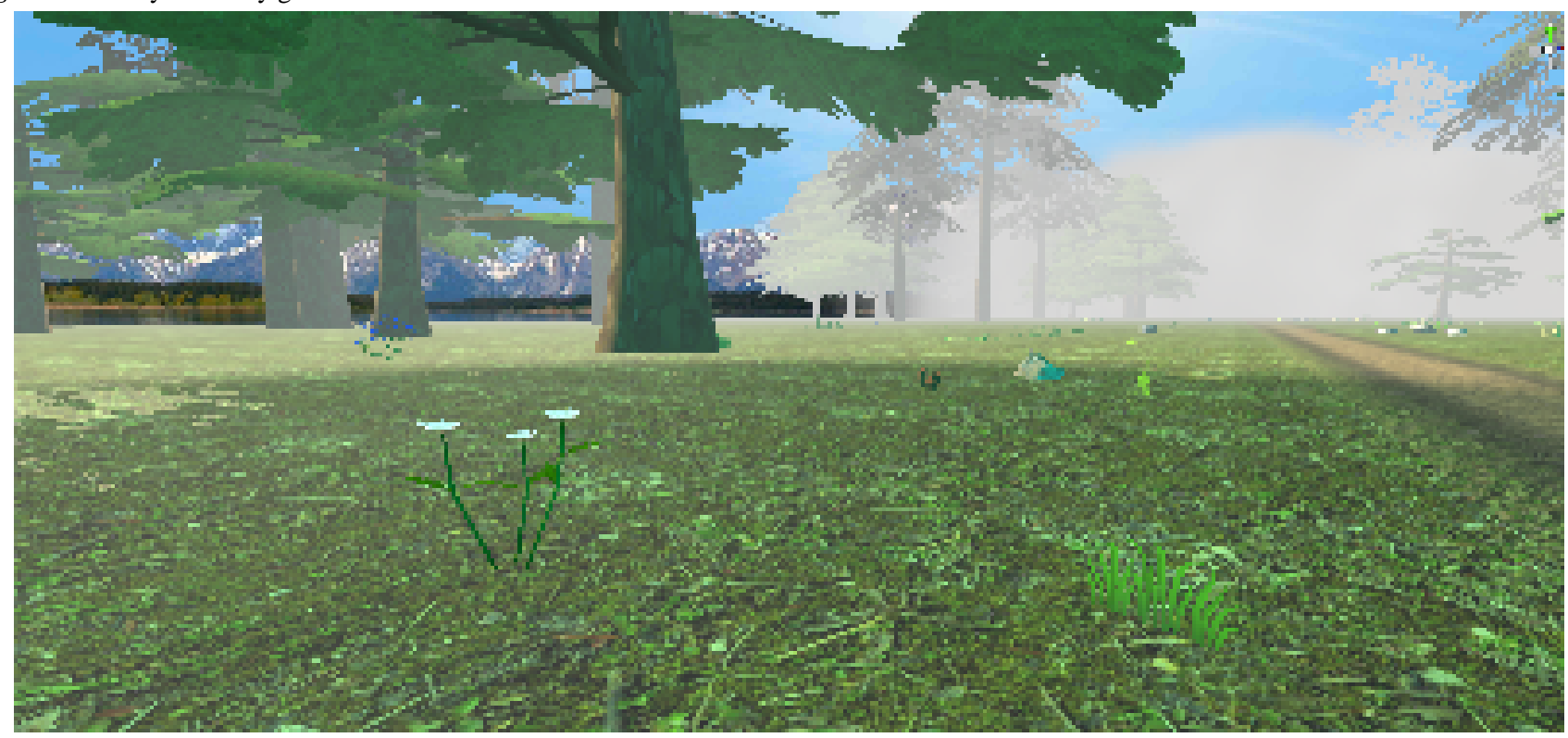

\section{Head-Mounted Display}

The VRADA app uses Oculus Go as the main VR head-mounted display (Figure 5). Oculus Go is a 3DOF (degrees of freedom) headset with a single 3DOF controller. As a standalone untethered headset, it does not need a desktop connection, and the absence of cables makes the device mobile and ideal for use while exercising. 
Figure 5. The Oculus Go Head-Mounted display.

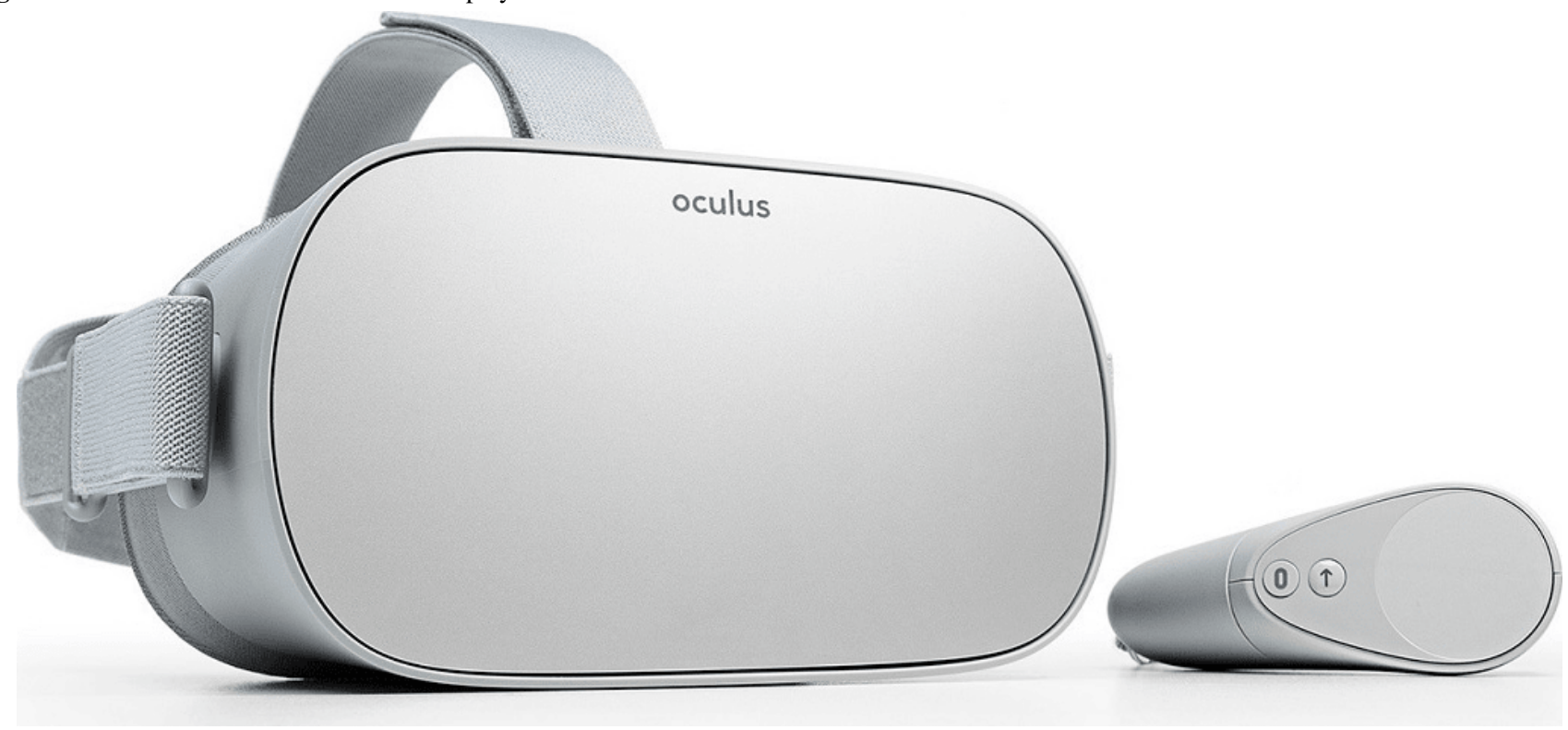

\section{Feasibility Studies VR2a and VR2b}

When the development of the first VRADA prototype reached a satisfactory level, we conducted an early feasibility trial, focusing on acceptability, usability, and tolerability, first with university students and then with the intended end users (Alzheimer Hellas for Alzheimer Association). The efficacy of the final version of the system will be tested in a future RCT (VR3).

Tables 2 and 3 presents the means, SD, Cronbach $\alpha$, and Pearson correlations for Studies VR2a and VR2b. All scales from both studies exhibited high internal consistency (Cronbach $\alpha .71$ and .89), with the exception of the SUS, which returned lower (.67 and .68 for VR2a and VR2b, respectively) but still acceptable internal consistency [71]. 
Table 2. Descriptive statistics for study VR2a, including Cronbach $\alpha$ and Pearson correlations.

\begin{tabular}{|c|c|c|c|c|c|c|c|c|c|c|}
\hline \multirow[t]{2}{*}{ Variables } & \multicolumn{8}{|c|}{ Study VR2a } & \multirow[t]{2}{*}{ Mean (SD) } & \multirow[t]{2}{*}{ Cronbach $\alpha$} \\
\hline & $\mathrm{PI}^{\mathrm{a}}$ & $\mathrm{PE}^{\mathrm{b}}$ & $\mathrm{ITU}^{\mathrm{c}}$ & $\mathrm{ATT}^{\mathrm{d}}$ & SUS ${ }^{\mathrm{e}}$ & PREF $^{f}$ & $\mathrm{UP}^{\mathrm{g}}$ & $\mathrm{UL}^{\mathrm{h}}$ & & \\
\hline PI & & & & & & & & & $3.68(0.65)$ & .76 \\
\hline$r$ & $-\mathrm{i}$ & & & & & & & & & \\
\hline$P$ value & - & & & & & & & & & \\
\hline $\mathbf{P E}$ & & & & & & & & & $4.22(0.61)$ & .88 \\
\hline$r$ & 0.14 & - & & & & & & & & \\
\hline$P$ value & .45 & - & & & & & & & & \\
\hline ITU & & & & & & & & & $3.91(0.95)$ & .89 \\
\hline$r$ & 0.18 & 0.78 & - & & & & & & & \\
\hline$P$ value & .34 & $<.001$ & - & & & & & & & \\
\hline ATT & & & & & & & & & $6.12(0.78)$ & .79 \\
\hline$r$ & -0.00 & 0.67 & 0.53 & - & & & & & & \\
\hline$P$ value & .96 & $<.001$ & $<.001$ & - & & & & & & \\
\hline SUS & & & & & & & & & $82.66(9.00)$ & .67 \\
\hline$r$ & 0.13 & 0.48 & 0.24 & 0.47 & - & & & & & \\
\hline$P$ value & .49 & $<.001$ & .19 & $<.001$ & - & & & & & \\
\hline PREF & & & & & & & & & $0.65(0.41)$ & .71 \\
\hline$r$ & 0.00 & 0.28 & 0.44 & 0.24 & 0.10 & - & & & & \\
\hline$P$ value & .96 & .13 & .01 & .19 & .58 & - & & & & \\
\hline UP & & & & & & & & & $4.33(0.58)$ & .58 \\
\hline$r$ & 0.31 & 0.54 & 0.28 & 0.35 & 0.52 & -0.04 & - & & & \\
\hline$P$ value & .08 & $<.001$ & .12 & .05 & $<.001$ & .83 & - & & & \\
\hline UL & & & & & & & & & $4.40(0.66)$ & .59 \\
\hline$r$ & 0.21 & 0.46 & 0.20 & 0.43 & 0.59 & 0.12 & 0.43 & - & & \\
\hline$P$ value & .25 & .01 & .28 & .01 & $<.001$ & .51 & .01 & - & & \\
\hline TOL $^{\mathbf{j}}$ & & & & & & & & & $3.94(0.89)$ & .61 \\
\hline$r$ & 0.19 & 0.41 & 0.42 & 0.27 & 0.60 & 0.51 & 0.56 & 0.34 & & \\
\hline$P$ value & .30 & .02 & .02 & .14 & $<.001$ & $<.001$ & $<.001$ & $<.001$ & & \\
\hline
\end{tabular}

${ }^{\mathrm{a}} \mathrm{PI}$ : personal innovativeness.

${ }^{b}$ PE: perceived enjoyment.

${ }^{\mathrm{c}}$ ITU: intention to use.

${ }^{\mathrm{d}}$ ATT: attitudes.

${ }^{\text {e }}$ SUS: system usability scale.

${ }^{f}$ PREF: preferences.

${ }^{\mathrm{g}} \mathrm{UP}$ : usability-pleasantness.

${ }^{\mathrm{h}}$ UL: usability-learning.

${ }^{\mathrm{i}}$ Not applicable.

${ }^{\mathrm{j}}$ TOL: tolerability. 
Table 3. Descriptive statistics for study VR2b, including Cronbach $\alpha$ and Pearson correlations.

\begin{tabular}{|c|c|c|c|c|c|c|c|c|c|c|}
\hline \multirow[t]{2}{*}{ Variables } & \multicolumn{8}{|c|}{ Study VR2b } & \multirow[t]{2}{*}{ Mean (SD) } & \multirow[t]{2}{*}{ Cronbach $\alpha$} \\
\hline & $\mathrm{PI}^{\mathrm{a}}$ & $\mathrm{PE}^{\mathrm{b}}$ & $\mathrm{ITU}^{\mathrm{c}}$ & $\mathrm{ATT}^{\mathrm{d}}$ & SUS $^{\mathrm{e}}$ & PREF $^{\mathrm{f}}$ & $U P^{g}$ & $\mathrm{UL}^{\mathrm{h}}$ & & \\
\hline PI & & & & & & & & & $3.86(0.82)$ & .87 \\
\hline$r$ & $-\mathrm{i}$ & & & & & & & & & \\
\hline$P$ value & - & & & & & & & & & \\
\hline $\mathbf{P E}$ & & & & & & & & & $4.43(0.57)$ & .81 \\
\hline$r$ & 0.36 & - & & & & & & & & \\
\hline$P$ value & .06 & - & & & & & & & & \\
\hline ITU & & & & & & & & & $4.19(0.78)$ & .95 \\
\hline$r$ & 0.44 & 0.82 & - & & & & & & & \\
\hline$P$ value & .02 & $<.001$ & - & & & & & & & \\
\hline ATT & & & & & & & & & $6.17(1.00)$ & .74 \\
\hline$r$ & 0.05 & 0.47 & 0.34 & - & & & & & & \\
\hline$P$ value & .79 & .01 & .07 & - & & & & & & \\
\hline SUS & & & & & & & & & $77.96(13.40)$ & .68 \\
\hline$r$ & 0.48 & 0.68 & 0.70 & 0.43 & - & & & & & \\
\hline$P$ value & .01 & $<.001$ & $<.001$ & .02 & - & & & & & \\
\hline PREF & & & & & & & & & $0.72(0.51)$ & .89 \\
\hline$r$ & 0.06 & 0.23 & 0.22 & 0.37 & 0.10 & - & & & & \\
\hline$P$ value & .73 & .24 & .26 & .05 & .61 & - & & & & \\
\hline UP & & & & & & & & & $4.77(0.52)$ & .73 \\
\hline$r$ & 0.02 & 0.51 & 0.42 & 0.38 & 0.37 & 0.52 & - & & & \\
\hline$P$ value & .89 & .006 & .02 & .05 & .05 & $<.001$ & - & & & \\
\hline UL & & & & & & & & & $4.53(0.67)$ & .94 \\
\hline$r$ & 0.00 & 0.27 & 0.13 & 0.43 & 0.28 & 0.41 & 0.59 & - & & \\
\hline$P$ value & .99 & .16 & .51 & .02 & .15 & .03 & $<.001$ & - & & \\
\hline TOL $^{\mathbf{j}}$ & & & & & & & & & $4.26(0.96)$ & .89 \\
\hline$r$ & 0.13 & 0.26 & 0.36 & 0.48 & 0.47 & 0.38 & 0.53 & 0.33 & & \\
\hline$P$ value & .50 & .17 & .06 & .01 & .01 & .05 & .01 & .09 & & \\
\hline
\end{tabular}

${ }^{\mathrm{a}} \mathrm{PI}$ : personal innovativeness.

${ }^{b}$ PE: perceived enjoyment.

' ITU: intention to use.

${ }^{\mathrm{d}}$ ATT: attitudes.

${ }^{\mathrm{e}}$ SUS: system usability scale.

${ }^{f}$ PREF: preferences.

${ }^{\mathrm{g}} \mathrm{UP}$ : usability-pleasantness.

${ }^{\mathrm{h}} \mathrm{UL}$ : usability-learning.

${ }^{\mathrm{i}}$ Not applicable.

${ }^{\mathrm{j}}$ TOL: tolerability.

\section{Personal Innovativeness, Acceptability, and SUS}

In VR2a, students scored moderately to high on personal innovativeness and intended future use and high on attitudes toward VR exercise and enjoyment. The score for usability $(82.66 / 100)$ was well above the acceptability threshold (75/100).
In study VR2b, patients with MCI scored moderately to high on personal innovativeness and high on enjoyment, attitude to VR exercise, and intended future use. The usability score (77.96) was also above the acceptability threshold. 


\section{Preferences}

A single-sample $t$ test was performed to determine whether there was a statistically significant preference for either of the 2 conditions, coding the normal environment as -1 and the VR environment as +1 , with the test value set at 0 . For study VR2a, the analysis indicated a significant preference for the VR condition (mean 0.66, SD $0.41 ; \mathrm{t}_{29}=8.74 ; P<.001$ ). Similarly, for study VR2b, the analysis indicated a significant preference for the VR condition (mean 0.72 , SD $0.51 ; \mathrm{t}_{26}=7.36 ; P<.001$ ).

\section{Pearson Correlations}

Pearson correlations between all variables were calculated for the 2 studies (Tables 2 and 3). In study VR2a, innovativeness was unrelated to any of the other variables; acceptance variables were strongly interrelated and moderately related to usability. Finally, preference was most strongly related to intention. In study VR2b, innovativeness was moderately related to usability and to 2 acceptance variables (enjoyment and intention). Acceptance variables were positively interrelated and strongly related to usability. Finally, preference was most strongly related to attitude, but the relationship was not statistically significant.

\section{Evaluation of Headset, Controller, and VRADA Environment}

In study VR2a, students' scores were high for the VR gear, VRADA environment, preference, and usability and moderate to high for acceptance variables and innovativeness. In study VR2b, MCI patients' scores were high for the VR gear, VRADA environment, preference, and usability and moderate to high for acceptance variables and innovativeness.

The semistructured interview data regarding usability, sense of presence, tolerability, and expectations are summarized in Table 4. In both groups, most participants reported a preference for the VRADA training system compared with standard care training. Comments in relation to most dimensions of usability were also very similar in both groups, but the MCI patient group reported needing more help when learning how to use the VR equipment. The 2 groups differed in relation to perceived feeling of presence, but engagement and realism (as dimensions of sense of presence) exhibited the same direction. Surprisingly, tolerability was higher among the MCI patients, as was the intended future use of the system. 
Table 4. Summary of interview data: students and patients with mild cognitive impairment.

\begin{tabular}{ll}
\hline Main theme & Subthemes \\
& Study VR'2a (students) \\
\hline Reasons to use VRADAc & Because: \\
& - ...."VRADA is more pleasant and interesting" (51\%) \\
& - ...."itime passes faster" (31\%) \\
& - ..."it is less boring" $(10 \%)$ \\
&
\end{tabular}

Expectations

Tolerability

- $\quad$ Feel bad during training: No $85 \%$, Yes $15 \%$

- Feel nausea, dizziness, or other physical symptoms: No $62 \%$, Yes $38 \%$

- Need for extra help: No $100 \%$

- Need more time to understand the system: No $100 \%$

- Pleasantness:

- Most enjoyable parts (environment 68\%, music $32 \%$ )

- Least enjoyable parts (repeated virtual parts $48 \%$, graphics $37 \%$, music $15 \%$ )

- $\quad$ Feel uncomfortable: No 83\%, dizziness $17 \%$

- Realism:

- $\quad$ Realistic or artificial virtual environment (realistic $3 \%$, so-so $20 \%$, artificial $77 \%$ )
Study VR2b (patients with $\mathrm{MCI}^{\mathrm{b}}$ )

Because:

- ..."VRADA is more pleasant, beautiful, and interesting" $(56 \%)$

- ..."time passes faster" $(28 \%)$

- ..."it is like escaping from reality" $(12 \%)$

- ..."it improves visibility" (4\%)

- $\quad$ Future personal use of the system: Yes: $81 \%$; So-so: $12 \%$; No: $7 \%$

- System is useful for other populations (young $18 \%$, everybody $23 \%$, disabilities $41 \%$, people who like to explore nature $18 \%$ )

- Utilization:

- General difficulties (no difficulties 63\%, VR controller $23 \%$, speed $14 \%$ )

- $\quad$ Technical problems (none $87 \%$, connectivity $13 \%$ )

- VR controller use (OK 50\%, control and sensitivity $50 \%$ )

- $\quad$ VR mask use (OK 95\%, dysphoria 5\%)

- Learning to use:

- Learning to use:

- Need for extra help: No 78\%, Yes (how to start) $13 \%$; Yes (how to use VR controller) $9 \%$

- Need more time to understand the system: (No $88 \%$, Yes $12 \%$ )

- Pleasantness:

- Most enjoyable parts (environment 92\%, animals $8 \%$ )

- Least enjoyable parts (repeated virtual parts $75 \%$, VR controller $13 \%$, graphics $12 \%$ )

- Feel uncomfortable: No 95\%, VR mask 5\%

- Spatial presence:

- $\quad$ Feeling of presence: Yes $77 \%$, So-so $12 \%$, No $11 \%)$

- Control of the system: Yes $88 \%$, So-so $4 \%$, No $8 \%$

- Engagement:

- Duration of experience (prefer more 53\%, good $47 \%$ ), distraction of attention: No $76 \%$, VR controller $12 \%$, cognitive exercises $12 \%$

- Realism:

- Realistic or artificial virtual environment (realistic $34 \%$, artificial $66 \%$ )

- $\quad$ Feel bad during training (No 93\%, Yes 7\%)

- $\quad$ Feel nausea, dizziness, or other physical symptoms: No $93 \%$, Yes $7 \%$ )

${ }^{\mathrm{a}}$ VR: virtual reality.

${ }^{\mathrm{b}} \mathrm{MCI}$ : mild cognitive impairment.

${ }^{\mathrm{c}}$ VRADA: VR Exercise App for Dementia and Alzheimer's Patients. 


\section{Discussion}

\section{Principal Findings}

The development of the VRADA training system followed the latest proposed recommendations and strategies $[56,57]$ for VR therapeutic and health apps. In line with these guidelines, we focused on the development of the content, VR environment, and system architecture (VR1). In collaboration with patients and health care providers and on the basis of the principles of human-centered design and continuous testing, VRADA incorporated dual task cognitive and physical training for older patients with MCI in a user-friendly VR environment.

The quantitative and qualitative data (VR2) regarding the acceptability, usability, and tolerability of VRADA as a training system are encouraging. As evidenced by the scores across all acceptability dimensions, the system was well accepted by both test groups. Acceptability is a key issue for innovative technology in the treatment schemes of neurodegenerative diseases, especially among the older population [37,38,72,73]. Scores for perceived enjoyment, attitude, and intention to use were well above average, with end users scoring higher than the students' group on all dimensions. The MCI patient interview comments were also very encouraging regarding the future use of VRADA, as they found the system more pleasant and interesting. This positive attitude toward VR among older people aligns with previous research. One systematic review [74] reported evidence that technology is a well-accepted method to provide engaging exercise opportunities to older people, and the high adherence rates can be explained largely by the high reported levels of enjoyment they experience when using these programs.

The SUS results were also very encouraging, indicating that the system exhibited good usability. Developing a usable, immersive VR system for older people at risk of cognitive decline requires careful consideration, and our design followed the latest recommendations [75] for developing similar VR platforms. We involved an interdisciplinary group of experts from the early stages of development and tried to meet basic psychological needs [76] by promoting user autonomy by providing choices. We also addressed their need to feel competent by providing encouragement, feedback, and an easy-to-use system that contributed to enjoyment and satisfaction. As almost half of our older participants were relatively unfamiliar with novel technology use, we provided a short initial training element to familiarize them with VR. However, the end users' comments suggest a need to provide more detailed instructions on using the system; in particular, some additional time might be needed for them to become familiar with the controller.

Simulator sickness, which can be attributed to postural instability or sensory conflict, is often a concern when older people use immersive VR [77]. However, most participants from both groups $(93 \%)$ tolerated the VRADA training system very well, with no adverse effects (eg, nausea, dizziness, and anxiety) among the patients with MCI, who perceived it as an enjoyable experience. Finally, the correlation results show that personal innovativeness was unrelated to any of the other variables, indicating that the system may be attractive and interesting even for less innovation-seeking users, at least within this sample.

\section{Strengths and Limitations}

The VRADA training system is among the first to attempt to transfer standard care, nonpharmacological, cognitive, and physical training as a simultaneous dual task MCI treatment scheme to a virtual environment. According to the literature [78], direct and indirect interventions targeting cognitive-motor interference have shown promise as a means of improving MCI in individuals with neurodegenerative diseases. Although similar previous studies have reported encouraging results [37,38], the dual task training program was sequential rather than simultaneous. Moreover, VR technology training environments such as VRADA provide flexibility in clinical settings because they can be tailored to individual needs and facilitate training in settings that are either impossible or unsafe in the real world. VRADA may also help to increase older people's autonomy and has the potential to reduce the workload of health care professionals dealing with patients with MCI. Finally, VRADA can be combined with other techniques, such as functional magnetic resonance imaging, to track brain functionality in the VR environment. This can provide further valuable insights into the effects of VR [79].

Despite these positive results regarding acceptability, usability, and tolerability, this study has some limitations. As the VRADA training system was only tested in a single session, we cannot conclude that this training would remain interesting and enjoyable after repeated longer sessions, and future studies should explore this issue. It is also important to emphasize that this study was designed to test the feasibility of the VRADA training system but not its efficacy. We intend to test efficacy in the near future in an RCT that will examine whether regular training using the VRADA system results in improved physical, cognitive, and quality of life outcomes.

\section{Conclusions}

This study addressed the design of a user-friendly, acceptable, and tolerable immersive VR system for dual task physical and cognitive skills training. Both students and older people with MCI symptoms reported high levels of acceptability, usability, and tolerability when using the VRADA training system, confirming its potential (subject to RCT efficacy validation) as a tool to promote physical and cognitive health among patients with MCI.

\section{Acknowledgments}

This research is cofunded by the European Regional Development Fund of the EU and Greek national funds through the Operational Program Competitiveness, Entrepreneurship, and Innovation, under the call RESEARCH-CREATE-INNOVATE (project code: T1EDK-01448). The funding agency played no role in the design or execution of the study, analysis or interpretation of the data, or decision to submit the results. Data are available upon request from YT. 
The authors gratefully acknowledge the ongoing support from the Hellenic Association of Alzheimer and Related Disorders during the VRADA project.

\section{Conflicts of Interest}

None declared.

\section{Multimedia Appendix 1}

Baseline demographic characteristics: studies VR2a and VR2b.

[DOCX File, 31 KB-Multimedia Appendix 1]

\section{Multimedia Appendix 2}

Video of the virtual reality Exercise App for Dementia and Alzheimer's Patients training environment. [MP4 File (MP4 Video), 74185 KB-Multimedia Appendix 2]

\section{Multimedia Appendix 3}

Interview guide.

[DOCX File, 14 KB-Multimedia Appendix 3]

\section{References}

1. Arai H, Ouchi Y, Yokode M, Ito H, Uematsu H, Eto F, Members of Subcommittee for Aging. Toward the realization of a better aged society: messages from gerontology and geriatrics. Geriatr Gerontol Int 2012 Jan;12(1):16-22. [doi: 10.1111/j.1447-0594.2011.00776.x] [Medline: 22188494]

2. Dementia: Key facts. World Health Organization.: WHO URL: https://www.who.int/news-room/fact-sheets/detail/dementia [accessed 2021-02-20]

3. Gauthier S, Reisberg B, Zaudig M, Petersen RC, Ritchie K, Broich K, et al. Mild cognitive impairment. The Lancet 2006 Apr;367(9518):1262-1270. [doi: 10.1016/s0140-6736(06)68542-5]

4. Petersen RC, Lopez O, Armstrong MJ, Getchius TS, Ganguli M, Gloss D, et al. Practice guideline update summary: Mild cognitive impairment. Neurology 2017 Dec 27;90(3):126-135. [doi: 10.1212/wnl.0000000000004826]

5. Karssemeijer EGA, Aaronson JA, Bossers WJ, Smits T, Olde Rikkert MGM, Kessels RPC. Positive effects of combined cognitive and physical exercise training on cognitive function in older adults with mild cognitive impairment or dementia: A meta-analysis. Ageing Res Rev 2017 Nov;40:75-83. [doi: 10.1016/j.arr.2017.09.003] [Medline: 28912076]

6. Langa KM, Levine DA. The diagnosis and management of mild cognitive impairment: a clinical review. JAMA 2014 Dec 17;312(23):2551-2561 [FREE Full text] [doi: 10.1001/jama.2014.13806] [Medline: 25514304]

7. Panza GA, Taylor BA, MacDonald HV, Johnson BT, Zaleski AL, Livingston J, et al. Can Exercise Improve Cognitive Symptoms of Alzheimer's Disease? J Am Geriatr Soc 2018 Jan 24;66(3):487-495. [doi: 10.1111/jgs.15241]

8. Schneider LS, Mangialasche F, Andreasen N, Feldman H, Giacobini E, Jones R, et al. Clinical trials and late-stage drug development for Alzheimer's disease: an appraisal from 1984 to 2014. J Intern Med 2014 Mar 08;275(3):251-283. [doi: 10.1111/joim.12191]

9. Song D, Yu DS, Li PW, Lei Y. The effectiveness of physical exercise on cognitive and psychological outcomes in individuals with mild cognitive impairment: A systematic review and meta-analysis. International Journal of Nursing Studies 2018 Mar;79:155-164. [doi: 10.1016/j.ijnurstu.2018.01.002]

10. Wang Y, Jia R, Liang J, Li J, Qian S, Li J, et al. Effects of non-pharmacological therapies for people with mild cognitive impairment. A Bayesian network meta-analysis. Int J Geriatr Psychiatry 2020 Jun 12;35(6):591-600. [doi: 10.1002/gps.5289] [Medline: 32119152]

11. Couch E, Lawrence V, Co M, Prina M. Outcomes tested in non-pharmacological interventions in mild cognitive impairment and mild dementia: a scoping review. BMJ Open 2020 Apr 20;10(4):e035980 [FREE Full text] [doi:

10.1136/bmjopen-2019-035980] [Medline: 32317262]

12. Hertzog C, Kramer AF, Wilson RS, Lindenberger U. Enrichment Effects on Adult Cognitive Development: Can the Functional Capacity of Older Adults Be Preserved and Enhanced? Psychol Sci Public Interest 2008 Oct;9(1):1-65. [doi: 10.1111/j.1539-6053.2009.01034.x] [Medline: 26162004]

13. Gillig PM, Sanders RD. Psychiatry, neurology, and the role of the cerebellum. Psychiatry (Edgmont) 2010 Sep;7(9):38-43 [FREE Full text] [Medline: 20941351]

14. Nathaniel-James D. The Role of the Dorsolateral Prefrontal Cortex: Evidence from the Effects of Contextual Constraint in a Sentence Completion Task. NeuroImage 2002 Aug;16(4):1094-1102. [doi: 10.1006/nimg.2002.1167]

15. Wulf G, Prinz W. Directing attention to movement effects enhances learning: A review. Psychonomic Bulletin \& Review 2001 Dec;8(4):648-660. [doi: 10.3758/bf03196201] 
16. Olazaran J, Muniz R, Reisberg B, Pena-Casanova J, del Ser T, Cruz-Jentoft AJ, et al. Benefits of cognitive-motor intervention in MCI and mild to moderate Alzheimer disease. Neurology 2004 Dec 28;63(12):2348-2353. [doi: 10.1212/01.wnl.0000147478.03911.28]

17. Hillman CH, Erickson KI, Kramer AF. Be smart, exercise your heart: exercise effects on brain and cognition. Nat Rev Neurosci 2008 Jan;9(1):58-65. [doi: 10.1038/nrn2298]

18. Morris JC, Storandt M, Miller JP, McKeel DW, Price JL, Rubin EH, et al. Mild Cognitive Impairment Represents Early-Stage Alzheimer Disease. Arch Neurol 2001 Mar 01;58(3). [doi: 10.1001/archneur.58.3.397]

19. Tsolaki M, Kazis A. Dementia, Medicine and Social Challenge. Thessaloniki, Greece: University Studio Press; 2005.

20. Erickson KI, Voss MW, Prakash RS, Basak C, Szabo A, Chaddock L, et al. Exercise training increases size of hippocampus and improves memory. In: Proceedings of the National Academy of Sciences. 2011 Jan 31 Presented at: Proc Natl Acad Sci USA. ;108?22; 2011; USA p. 3017-3022. [doi: 10.1073/pnas.1015950108]

21. Lautenschlager NT, Cox KL, Flicker L, Foster JK, van BFM, Xiao J, et al. Effect of physical activity on cognitive function in older adults at risk for Alzheimer disease: a randomized trial. JAMA 2008 Sep 03;300(9):1027-1037. [doi: 10.1001/jama.300.9.1027] [Medline: 18768414]

22. Lauenroth A, Ioannidis AE, Teichmann B. Influence of combined physical and cognitive training on cognition: a systematic review. BMC Geriatr 2016 Jul 18;16(1). [doi: 10.1186/s12877-016-0315-1]

23. Kounti F, Bakoglidou E, Agogiatou C, Lombardo NBE, Serper LL, Tsolaki M. RHEA,* a Nonpharmacological Cognitive Training Intervention in Patients With Mild Cognitive Impairment. Topics in Geriatric Rehabilitation 2011;27(4):289-300. [doi: 10.1097/tgr.0b013e31821e59a9]

24. Montero-Odasso MM, Sarquis-Adamson Y, Speechley M, Borrie MJ, Hachinski VC, Wells J, et al. Association of Dual-Task Gait With Incident Dementia in Mild Cognitive Impairment. JAMA Neurol 2017 Jul 01;74(7):857. [doi: 10.1001/jamaneurol.2017.0643]

25. Tay L, Lim WS, Chan M, Ali N, Chong MS. A Combined Cognitive Stimulation and Physical Exercise Programme (MINDVital) in Early Dementia: Differential Effects on Single- and Dual-Task Gait Performance. Gerontology 2016 Feb 26;62(6):604-610. [doi: 10.1159/000444084]

26. Schwenk M, Zieschang T, Oster P, Hauer K. Dual-task performances can be improved in patients with dementia: A randomized controlled trial. Neurology 2010 May 05;74(24):1961-1968. [doi: 10.1212/wnl.0b013e3181e39696]

27. Ghai S, Ghai I, Effenberg AO. Effects of dual tasks and dual-task training on postural stability: a systematic review and meta-analysis. CIA 2017 Mar;Volume 12:557-577. [doi: 10.2147/cia.s125201]

28. Fritz NE, Cheek FM, Nichols-Larsen DS. Motor-Cognitive Dual-Task Training in Persons With Neurologic Disorders. Journal of Neurologic Physical Therapy 2015;39(3):142-153. [doi: 10.1097/npt.0000000000000090]

29. Wajda DA, Mirelman A, Hausdorff JM, Sosnoff JJ. Intervention modalities for targeting cognitive-motor interference in individuals with neurodegenerative disease: a systematic review. Expert Review of Neurotherapeutics 2016 Sep 12;17(3):251-261. [doi: 10.1080/14737175.2016.1227704]

30. D'Cunha NM, Nguyen D, Naumovski N, McKune AJ, Kellett J, Georgousopoulou EN, et al. A Mini-Review of Virtual Reality-Based Interventions to Promote Well-Being for People Living with Dementia and Mild Cognitive Impairment. Gerontology 2019;65(4):430-440 [FREE Full text] [doi: 10.1159/000500040] [Medline: 31108489]

31. Larson EB, Feigon M, Gagliardo P, Dvorkin AY. Virtual reality and cognitive rehabilitation: A review of current outcome research. NRE 2014 Jun 26;34(4):759-772. [doi: 10.3233/nre-141078]

32. Garcia L, Kartolo A, Méthot-Curtis E. A discussion of the use of virtual reality in dementia. In: Eichenberg C, editor. Virtual Reality in Psychological, Medical and Pedagogical Applications. New York, NY: USA: INTECH Open Access Publisher; 2012.

33. Tarnanas I, Tsolakis A, Tsolaki M. Assessing virtual reality environments as cognitive stimulation method for patients with MCI. In: Brooks A, Brahnam S, Jain L, editors. Technologies of Inclusive Well-Being. Berlin: Springer; 2014:39-74.

34. Liu Y, Tan W, Chen C, Liu C, Yang J, Zhang Y. A Review of the Application of Virtual Reality Technology in the Diagnosis and Treatment of Cognitive Impairment. Front. Aging Neurosci 2019 Oct 18;11. [doi: 10.3389/fnagi.2019.00280]

35. Kim O, Pang Y, Kim J. The effectiveness of virtual reality for people with mild cognitive impairment or dementia: a meta-analysis. BMC Psychiatry 2019 Jul 12;19(1). [doi: 10.1186/s12888-019-2180-x]

36. Ge S, Zhu Z, Wu B, McConnell ES. Technology-based cognitive training and rehabilitation interventions for individuals with mild cognitive impairment: a systematic review. BMC Geriatr 2018 Sep 15;18(1). [doi: 10.1186/s12877-018-0893-1]

37. Mrakic-Sposta S, Di Santo SG, Franchini F, Arlati S, Zangiacomi A, Greci L, et al. Effects of Combined Physical and Cognitive Virtual Reality-Based Training on Cognitive Impairment and Oxidative Stress in MCI Patients: A Pilot Study. Front Aging Neurosci 2018;10:282 [FREE Full text] [doi: 10.3389/fnagi.2018.00282] [Medline: $\underline{30327596]}$

38. Liao Y, Chen I, Lin Y, Chen Y, Hsu W. Effects of Virtual Reality-Based Physical and Cognitive Training on Executive Function and Dual-Task Gait Performance in Older Adults With Mild Cognitive Impairment: A Randomized Control Trial. Front Aging Neurosci 2019 Jul 16;11. [doi: 10.3389/fnagi.2019.00162]

39. Bamidis PD, Fissler P, Papageorgiou SG, Zilidou V, Konstantinidis EI, Billis AS, et al. Gains in cognition through combined cognitive and physical training: the role of training dosage and severity of neurocognitive disorder. Front Aging Neurosci 2015 Aug;7:152 [FREE Full text] [doi: 10.3389/fnagi.2015.00152] [Medline: 26300772] 
40. Lusk MM, Atkinson RK. Animated pedagogical agents: does their degree of embodiment impact learning from static or animated worked examples? Appl. Cognit. Psychol 2007 Sep;21(6):747-764. [doi: 10.1002/acp.1347]

41. Shapiro L. Embodied Cognition. New York: NY: Routledge; 2011.

42. Hung I, Lin L, Fang W, Chen N. Learning with the Body: An Embodiment-Based Learning Strategy Enhances Performance of Comprehending Fundamental Optics. Interacting with Computers 2014 Mar 26;26(4):360-371. [doi: 10.1093/iwc/iwu011]

43. Buzsáki G, Moser EI. Memory, navigation and theta rhythm in the hippocampal-entorhinal system. Nat Neurosci 2013 Jan 28;16(2):130-138. [doi: 10.1038/nn.3304]

44. Sauzéon H, N'Kaoua B, Arvind Pala P, Taillade M, Guitton P. Age and active navigation effects on episodic memory: A virtual reality study. Br J Psychol 2015 Feb 28;107(1):72-94. [doi: 10.1111/bjop.12123]

45. Sowndararajan A, Wang R, Bowman D. Quantifying the benefits of immersion for procedural training. : ACM; 2008 Aug Presented at: Workshop on Immersive Projection Technologies/Emerging Display Technologies, IPT/EDT August ;2, New York, USA, . ACM; 2008; New York, USA p. 1-2 URL: https://doi.org/10.1145/1394669.1394672 [doi: 10.1145/1394669.1394672]

46. Intraraprasit M, Phanpanya P, Jinjakam C. Cognitive training using immersive virtual reality. 2017 Presented at: 10 th Biomedical Engineering International Conference (BMEiCON); 2017; Hokkaido p. 1-5. [doi: 10.1109/bmeicon.2017.8229126]

47. Person-centred care. The key points of person-centred care. United Kingdom: Alzheimer's Society URL: https://www. alzheimers.org.uk/about-dementia/treatments/person-centred-care [accessed 2021-02-20]

48. Ryan RM, Deci EL. Self-determination theory and the facilitation of intrinsic motivation, social development, and well-being. American Psychologist 2000;55(1):68-78. [doi: 10.1037/0003-066X.55.1.68]

49. Ryan R, Deci E. Self-Determination Theory: Basic Psychological Needs in Motivation, Development, Wellness. New York, USA: Guilford; 2017.

50. Richard E, Schmand B, Eikelenboom P, Yang S, Ligthart S, Moll van Charante E, et al. Symptoms of Apathy Are Associated with Progression from Mild Cognitive Impairment to Alzheimer's Disease in Non-Depressed Subjects. Dement Geriatr Cogn Disord 2012;33(2-3):204-209. [doi: 10.1159/000338239]

51. Lanctôt KL, Agüera-Ortiz L, Brodaty H, Francis PT, Geda YE, Ismail Z, et al. Apathy associated with neurocognitive disorders: Recent progress and future directions. Alzheimer's \& Dementia 2016 Jun 27;13(1):84-100. [doi: 10.1016/j.jalz.2016.05.008]

52. Michie S, Richardson M, Johnston M, Abraham C, Francis J, Hardeman W, et al. The behavior change technique taxonomy (v1) of 93 hierarchically clustered techniques: building an international consensus for the reporting of behavior change interventions. Ann Behav Med 2013 Aug;46(1):81-95. [doi: 10.1007/s12160-013-9486-6] [Medline: 23512568]

53. Teixeira PJ, Marques MM, Silva MN, Brunet J, Duda JL, Haerens L, et al. A classification of motivation and behavior change techniques used in self-determination theory-based interventions in health contexts. Motivation Science 2020 Mar 05. [doi: $10.1037 / \operatorname{mot} 0000172]$

54. Knittle K, Heino M, Marques MM, Stenius M, Beattie M, Ehbrecht F, et al. The compendium of self-enactable techniques to change and self-manage motivation and behaviour v.1.0. Nat Hum Behav 2020 Jan 13;4(2):215-223. [doi: 10.1038/s41562-019-0798-9]

55. Jerald J. The VR Book: Human-Centered Design for Virtual Reality. New York, USA: Morgan and Claypool; 2016.

56. Mummah SA, Robinson TN, King AC, Gardner CD, Sutton S. IDEAS (Integrate, Design, Assess, and Share): A Framework and Toolkit of Strategies for the Development of More Effective Digital Interventions to Change Health Behavior. J Med Internet Res 2016 Dec 16;18(12):e317 [FREE Full text] [doi: 10.2196/jmir.5927] [Medline: 27986647]

57. Birckhead B, Khalil C, Liu X, Conovitz S, Rizzo A, Danovitch I, et al. Recommendations for Methodology of Virtual Reality Clinical Trials in Health Care by an International Working Group: Iterative Study. JMIR Ment Health 2019 Jan 31;6(1):e11973. [doi: 10.2196/11973]

58. Petersen RC, Morris JC. Mild Cognitive Impairment as a Clinical Entity and Treatment Target. Arch Neurol 2005 Jul 01;62(7):1160. [doi: 10.1001/archneur.62.7.1160]

59. Reisberg B, Ferris SH, de Leon MJ, Crook T. The Global Deterioration Scale for assessment of primary degenerative dementia. Am J Psychiatry 1982 Sep;139(9):1136-1139. [doi: 10.1176/ajp.139.9.1136] [Medline: 7114305]

60. Morris JC. The Clinical Dementia Rating (CDR): Current version and scoring rules. Neurology 1993 Nov 01;43(11):2412-2412. [doi: 10.1212/WNL.43.11.2412-a]

61. Jessen F, Amariglio RE, van Boxtel M, Breteler M, Ceccaldi M, Chételat G, et al. A conceptual framework for research on subjective cognitive decline in preclinical Alzheimer's disease. Alzheimer's \& Dementia 2014 May 02;10(6):844-852. [doi: $10.1016 /$ j.jalz.2014.01.001]

62. Agarwal R, Prasad J. A Conceptual and Operational Definition of Personal Innovativeness in the Domain of Information Technology. Information Systems Research 1998 Jun;9(2):204-215. [doi: 10.1287/isre.9.2.204]

63. Rasimah CMY, Ahmad A, Zaman HB. Evaluation of user acceptance of mixed reality technology. AJET 2011 Dec 23;27(8). [doi: 10.14742/ajet.899]

64. Francis J, Eccles MP, Johnston M, Walker AE, Grimshaw JM, Foy R, et al. Constructing questionnaires based on the theory of planned behaviour: A manual for health services researchers. City, University of London Institutional Repository. 
Newcastle upon Tyne, UK: Centre for Health Services Research, University of Newcastle upon Tyne; 2004. URL: http:/ /openaccess.city.ac.uk/1735/1/TPB\%20Manual\%20FINAL\%20May2004.pdf [accessed 2021-03-18]

65. Ajzen I. Constructing a theory of planned behavior questionnaire. https://people.umass.edu/aizen/. URL: https://people. umass.edu/aizen/pdf/tpb.measurement.pdf [accessed 2021-02-20]

66. Brooke J. SUS - A quick and dirty usability scale. In: Jordan PW, Thomas B, McClelland IL, Weerdmeester B, editors. Usability Evaluation in Industry. Bristol: Taylor \& Francis Ltd; 1996:189-194.

67. Pedroli E, Greci L, Colombo D, Serino S, Cipresso P, Arlati S, et al. Characteristics, Usability, and Users Experience of a System Combining Cognitive and Physical Therapy in a Virtual Environment: Positive Bike. Sensors 2018 Jul 19;18(7):2343. [doi: $10.3390 / \mathrm{s} 18072343$ ]

68. Braun V, Clarke V. Using thematic analysis in psychology. Qualitative Research in Psychology 2006 Jan;3(2):77-101. [doi: 10.1191/1478088706qp063oa]

69. Papagiannakis G, Lydatakis N, Kateros S, Georgiou S, Zikas. Transforming medical educationtraining with VR using MAGES. SIGGRAPH Asia 2018 Posters (SA ?18). Association for Computing Machinery, New York, NY, USA. Article 83 2018:1. [doi: 10.1145/3283289.3283291]

70. Papagiannakis G, Zikas P, Lydatakis N. MAGES 3.0: tying the knot of medical VR. Eprint: 2005.01180, arXiv 2020:2005. [doi: $10.1145 / 3388536.3407888$ ]

71. Aiken, LR. Personality Assessment: Methods and Practices. 2nd Edition. Seattle, WA: Hogrefe \& Huber; 1996.

72. Dillon A, Morris M. User acceptance of new information technology: theories and models. In: Williams M, editor. Annual Review of Information Science and Technology. Medford NJ: Information Today; 1996:31-32.

73. D'Cunha NM, Isbel ST, Frost J, Fearon A, McKune AJ, Naumovski N, et al. Effects of a virtual group cycling experience on people living with dementia: A mixed method pilot study. Dementia (London) 2020 Aug 21:1471301220951328. [doi: 10.1177/1471301220951328] [Medline: 32820955]

74. Valenzuela T, Okubo Y, Woodbury A, Lord SR, Delbaere K. Adherence to Technology-Based Exercise Programs in Older Adults: A Systematic Review. J Geriatr Phys Ther 2016 Jun 29. [doi: 10.1519/JPT.0000000000000095] [Medline: 27362526]

75. Ijaz K, Ahmadpour N, Naismith SL, Calvo RA. An Immersive Virtual Reality Platform for Assessing Spatial Navigation Memory in Predementia Screening: Feasibility and Usability Study. JMIR Ment Health 2019 Sep 03;6(9):e13887. [doi: 10.2196/13887]

76. Peters D, Calvo RA, Ryan RM. Designing for Motivation, Engagement and Wellbeing in Digital Experience. Front Psychol 2018 May;9:797. [doi: 10.3389/fpsyg.2018.00797] [Medline: 29892246]

77. Cobb SVG, Nichols S, Ramsey A, Wilson JR. Virtual Reality-Induced Symptoms and Effects (VRISE). Presence: Teleoperators and Virtual Environments 1999 Apr;8(2):169-186. [doi: 10.1162/105474699566152]

78. Wajda DA, Mirelman A, Hausdorff JM, Sosnoff JJ. Intervention modalities for targeting cognitive-motor interference in individuals with neurodegenerative disease: a systematic review. Expert Review of Neurotherapeutics 2016 Sep 12;17(3):251-261. [doi: 10.1080/14737175.2016.1227704]

79. Bauer A, Andringa G. The Potential of Immersive Virtual Reality for Cognitive Training in Elderly. Gerontology 2020 Sep 9;66(6):614-623 [FREE Full text] [doi: 10.1159/000509830] [Medline: 32906122]

\section{Abbreviations}

AD: Alzheimer disease

DOF: degrees of freedom

EU: European Union

MCI: mild cognitive impairment

RCT: randomized controlled trial

SUS: system usability scale

VR: virtual reality

VRADA: VR Exercise App for Dementia and Alzheimer's Patients

WHO: World Health Organization 
Edited by G Eysenbach; submitted 08.09.20; peer-reviewed by N Martin, T Valenzuela; comments to author 17.10.20; revised version received 10.12.20; accepted 13.01.21; published 24.03 .21

Please cite as:

Hassandra M, Galanis E, Hatzigeorgiadis A, Goudas M, Mouzakidis C, Karathanasi EM, Petridou N, Tsolaki M, Zikas P, Evangelou G, Papagiannakis G, Bellis G, Kokkotis C, Panagiotopoulos SR, Giakas G, Theodorakis $Y$

A Virtual Reality App for Physical and Cognitive Training of Older People With Mild Cognitive Impairment: Mixed Methods Feasibility Study

JMIR Serious Games 2021;9(1):e24170

URL: https://games.jmir.org/2021/1/e24170

doi: $\underline{10.2196 / 24170}$

PMID: 33759797

(CMary Hassandra, Evangelos Galanis, Antonis Hatzigeorgiadis, Marios Goudas, Christos Mouzakidis, Eleni Maria Karathanasi, Niki Petridou, Magda Tsolaki, Paul Zikas, Giannis Evangelou, George Papagiannakis, George Bellis, Christos Kokkotis, Spyridon Rafail Panagiotopoulos, Giannis Giakas, Yannis Theodorakis. Originally published in JMIR Serious Games (http://games.jmir.org), 24.03.2021. This is an open-access article distributed under the terms of the Creative Commons Attribution License (https://creativecommons.org/licenses/by/4.0/), which permits unrestricted use, distribution, and reproduction in any medium, provided the original work, first published in JMIR Serious Games, is properly cited. The complete bibliographic information, a link to the original publication on http://games.jmir.org, as well as this copyright and license information must be included. 